\title{
Erste Systematisierungsansätze für die Beschreibung eines modellhaften \\ Crowdsourcing-Systems im Zusammenhang mit der Steuerung von Crowdsourcing
}

\author{
Marco Wedel und Hannah Ulbrich
}

\section{Zusammenfassung}

In der aktuellen Forschungsdiskussion sind grundlegende Fragestellungen zur Steuerung von Crowdsourcing, hier auch internes Crowdsourcing (IC), in Verbindung mit einer systematischen IC-Beschreibung weitgehend unbeantwortet. Dies wurde im Verlauf des Forschungsvorhabens „ICU - Internes Crowdsourcing in Unternehmen“ deutlich. Die Forschungslücke zur IC-Systematik wird an der begrifflichen Unschärfe der vorhandenen Beschreibungen von Steuerungsaktivitäten in Zusammenhang mit Crowdsourcing gegenständlich. So werden etwa Begriffe wie Governance und Prozessmanagement als Steuerungskategorien angeführt, zuweilen aber gleichbedeutend verwendet. Das Fehlen definitorischer Klarheit ist angesichts des relativ jungen IC-Phänomens nicht verwunderlich. Aufgrund der wachsenden (wissenschaftlichen) Popularität ist es jedoch notwendig, systemtheoretisierende Grundlegungen für IC zu erarbeiten. Am Beispiel der Beschreibung von Steuerungsaktivitäten wird in diesem Beitrag ein erster Aufschlag zur Erarbeitung systemtheoretisierender Grundlagen vorgenommen.

\section{$11.1 \quad$ Einleitung}

In der aktuellen Forschungsdiskussion sind grundlegende Fragestellungen zur Steuerung von Crowdsourcing, hier auch internes Crowdsourcing (IC), in Verbindung mit einer systematischen IC-Beschreibung noch weitgehend unbeantwortet. Dies wurde im Verlauf des

M. Wedel $(\bowtie) \cdot$ H. Ulbrich

Lehrstuhl für Arbeitslehre/Technik und Partizipation, Technische Universität Berlin,

Berlin, Deutschland

E-Mail: marco.wedel@tu-berlin.de; hannah.ulbrich@tu-berlin.de 
Forschungsvorhabens „ICU - Internes Crowdsourcing in Unternehmen“ deutlich. Das Ziel von ICU ist die Entwicklung eines in der Praxis getesteten, arbeitnehmerfreundlichen internen Crowdsourcing-Modells, das als Referenzfall guter Praxis für zukünftige Crowdsourcingaktivitäten dient. Im Fokus der Modellausgestaltung stehen die arbeitnehmergerechte Gestaltung der Anwendung, die digitale Beteiligung von Mitarbeiterinnen und Mitarbeitern an Unternehmensprozessen und die Eröffnung von Kompetenzerweiterungen durch Crowdarbeit. Bei IC erzeugen Beschäftigte eines Unternehmens (die Crowd) im Austausch über eine digitale Plattform Ideen und Lösungen, die zur Verbesserung von bestehenden Produkten, Prozessen und Dienstleistungen oder deren Neuentwicklungen (Innovationen) beitragen. IC stellt somit eine neue, digitale Form von innerbetrieblicher Wissensvernetzung und bereichsübergreifender Zusammenarbeit dar.

Grundsätzlich gibt es in Bezug auf systemtheoretisierende (nicht ausschließlich deskriptive) Auseinandersetzungen mit dem Thema Crowdsourcing praktisch keine belastbare Literatur. Es besteht hier die Notwendigkeit zur wissenschaftlichen Fundierung einer IC-Systematik mit verbindlichen Beschreibungskategorien. Das Fehlen definitorischer Klarheit ist angesichts des relativ jungen IC-Phänomens nicht verwunderlich. Aufgrund der wachsenden (wissenschaftlichen) Popularität ist es jedoch notwendig, systemtheoretisierende Grundlegungen für IC zu erarbeiten, um ein unverbindliches, schlimmstenfalls selbstreferenzielles, Nebeneinander eines rasant wachsenden Forschungsbestandes zu vermeiden. Da sich das Phänomen einer eindeutigen fachwissenschaftlichen Zuordnung entzieht, scheint es angebracht, interdisziplinäre Zugänge zu wählen und - ohne das Rad neu erfinden zu müssen - auf vorhandenen Theorie- und Terminologieangeboten aufzubauen.

Die Forschungslücke zur IC-Systematik wird deutlich in der begrifflichen Unschärfe der vorhandenen Beschreibungen von Steuerungsaktivitäten in Zusammenhang mit Crowdsourcing. So werden etwa Begriffe wie Governance und Prozessmanagement als Steuerungskategorien angeführt, zuweilen aber gleichbedeutend verwendet. Weitere systemtheoretische Zugänge, wie etwa Soziotechnische Systemtheorie (STS), werden in gleichem Zusammenhang in die Diskussion vermischend eingebracht (Blohm et al. 2018; Knop et al. 2017). Damit werden unter teils synonymer Verwendung von Steuerungs-, Governance- oder allgemeinen Systembeschreibungen so unterschiedliche Dinge adressiert wie Rollen-, Aufgaben-, Struktur- und Technologiebeschreibungen, Rahmenbedingungen, generell Mechanismen oder Aufgabenzuweisungen, weiterhin auch Aufgabendefinitionen, Aufgabentypen, Bewertungsmechanismen, Qualifizierungs- und Incentivierungsmechanismen sowie allgemeine Regelungen und Vereinbarungen (Alam und Campbell 2013; Blohm et al. 2018; Knop et al. 2017; Zogaj und Bretschneider 2014; Zuchowski et al. 2016).

Zwar kann und sollte eine verbindliche Systembeschreibung zur IC-Steuerung diese Teilbereiche adressieren und regeln, die einzelnen Teildimensionen selbst können aber nicht zum hinreichenden Beschreibungsmerkmal des Ganzen, hier einer Systematik IC-Steuerung bzw. Governance, werden. Für einen zukünftigen und zielführenden (Wissenschafts-) Diskurs gilt es, die Teilkategorien und Aspekte eines IC Systems sinnvoll zu referenzieren und in ein ordnendes Gesamtverhältnis zu setzten. 
Die Übergeordnete Fragestellung lautet entsprechend: (Wie) Können die bereits beschriebenen Teilkategorien und Aspekte eines IC-Systems sinnvoll referenziert und in ein ordnendes Gesamtverhältnis gesetzt werden? Welche Ergänzungen müssen für die Theoriebildung ggf. vorgenommen werden?

Der vorliegende Beitrag konzentriert sich dabei insbesondere auf die Identifikation von Beschreibungen und Definitionsangeboten in Zusammenhang mit Systematisierungsansätzen für die Entwicklung eines modellhaften Crowdsourcing-Systems im Zusammenhang mit der Steuerung von Crowdsourcing-Aktivitäten. Schon in den ersten wissenschaftlichen und wissenschaftsnahen Beschreibungsansätzen wird dabei deutlich, dass die definitionsunabhängige Anwendung bereits vorhandener Terminologieangebote anderer Wissenschaftsdisziplinen eine systemtheoretisierende Grundlegung für IC erschwert. Dies, so wird zu zeigen sein, drückt sich exemplarisch u. a. in der Anwendung des Governance-Begriffs zur Beschreibung von Steuerungsprinzipien bei Crowdsourcing aus.

\subsection{Beschreibungsansätze und Steuerungsprinzipien von Crowdsourcing}

Ansätze zur Beschreibung von systemischen Strukturen finden ihren Ausgangspunkt zumeist in der Analyse von identifizierbaren Rahmenbedingungen, die als solche einen ersten Hinweis auf Phänomen-immanente Charakteristika geben. Im Falle von IC haben Pedersen et al. (2013) in einer Literaturanalyse des Wissensbestandes im Hinblick auf ersten konzeptionellen Grundlegungen für Crowdsourcing die Elemente „Problem“, „People“, „Governance“, „Process“, „Technology“ und „Outcome“ als relevante Kategorien zur wissenschaftlichen Beschreibung und Analyse von Crowdsourcing identifiziert. In einer Literaturanalyse $\mathrm{zu}$ internem Crowdsourcing übernehmen und verfestigen Zuchowski et al. (2016, S. 168 f.) diese Sechs-Komponenten-Logik zur Beschreibung von identifizierbaren Rahmenbedingungen. In leicht veränderte Reihenfolge werden hier die ,problems component“, ,governance component“, ,,people commponent“, „IT component“, ,,process component“ und „outcome component“ zum hinreichenden Beschreibungsmerkmal eines grundsätzlichen IC-Rahmens (Zuchowski et al. 2016, ebd.).

Kurz umrissen adressiert die ,problems component“ die Frage, welche Probleme durch Crowdsourcing gelöst und welche Komplexitäts- und Differenzierungsgrade in einem Crowdsourcing-Verfahren aufgegriffen, berücksichtigt und bearbeitet werden können. Die „governance component“ verweist laut Pedersen et al. (2013, S. 582) grundsätzlich auf eine Steuerungsproblematik unter der Prämisse der Erreichung gewünschter Ziele, laut Zuchowski et al. (2016, S. 169) werden mit Governance allgemeine Managementaufgaben beschrieben. Unter „Process“ verstehen Pedersen et al. (2013, S. 581) eine Reihe von Maßnahmen, die von allen Beteiligten eines Crowdsourcing-Projektes durchgeführt werden müssen, um ein bestimmtes Problem zu lösen bzw. Ziel zu erreichen. Laut Zuchowski et al. (2016, S. 169) lässt sich der Prozess in die Phasen „Vorbereitung“, „Durchführung“, 
„Auswertung/Evaluation“ und „Lösung/Beschluss“ unterteilen. Mit dem Element „People" werden Rollen und Rollenmodelle und soziale Bedingungen zur Durchführung von Crowdsourcing-Aktivitäten adressiert. Unter der Überschrift „Technology“ bzw. „IT“ werden informationstechnologische Bedingungen für Crowdsourcing behandelt. Das Element „Outcome“ ist das letzte Modellelement der vorgeschlagenen Konzeption. Hier werden alle Aspekte die Ergebnisse des Crowdsourcing-Prozesses betreffend eingeordnet (Pedersen et al. 2013, S. 582 ff.; Zuchowski et al. 2016, S. 168 f.).

Knop et al. (2017) greifen die zuvor beschriebenen Elemente auf und ordnen diese aus der Theorieperspektive soziotechnischer Systeme. Der Ordnungsanspruch folgt hierbei der von Baxter und Sommerville (2011) skizzierten Prämisse, wonach das zu beschreibende System einen Prozess widerspiegelt, der sowohl soziale als auch technische Faktoren berücksichtigt, die jeweils die Funktionalität und Nutzung von IT-basierten Systemen originär beeinflussen. Beese et al. (2015) weisen in diesem Zusammenhang auf die enorme Komplexität soziotechnischer Systeme hin, die von einer Vielzahl von oft nichtlinearen und dynamischen Mechanismen abhängt, die sich sowohl auf die sozialen als auch auf die technischen Teilsysteme beziehen (Knop et al. 2017, S. 2 f.). In dieser Perspektive wird IC als soziotechnisches System beschrieben, das sich in die fünf Komponenten „Actors“, „Task“, „Structure“, „Technology“ und „Environment“ unterteilen lässt (Knop et al. 2017, S. 3).

Während sich die Elemente „Technology/IT“, „People/Actors“ und „Problem/Task“ damit in allen vorgeschlagenen Funktionsbeschreibungen mehr oder weniger deckungsgleich wiederfinden, bzw. eine solche Übereinstimmung an dieser Stelle vermutet wird (Pedersen et al. 2013; Zuchowski et al. 2016), verzichten Knop et al. (2017) auf die Elemente „Outcome“, „Process“ und „Governance“, führen ergänzend jedoch die Elemente „Environment“ und „Structure“ ein. Knop et al. (2017, S. 3) definieren das Element „Structure" als

systems of communication, systems of authority, and systems of workflow. It further includes both the normative dimension, that is, values, norms, and general role expectations, and the behavioral dimension, that is, the patterns of behavior as actors communicate, exercise authority, or work within the internal crowd.

Es sei an dieser Stelle argumentiert, dass das Element „Structure“, insoweit weitergehende und ausdifferenzierende Beschreibungen der hier vorgestellten Kategorien noch ausstehen, die Überlegungen von Pedersen et al. (2013) und Zuchowski et al. (2016) in Bezug auf „Process“ und „Governance“ inhaltlich-überschreibend zusammenführt. Eine Erklärung bzw. ein expliziter Verweis auf die Letztgenannten in Bezug auf die Erweiterung bzw. Veränderung der gewählten Kategorien durch Knop et al. (2017) konnte indes nicht gefunden werden.

Bei allen Unterschieden wird in den oben skizzierten Ansätzen zur Beschreibung eines IC-Systems der Versuch einer ersten funktionalen Differenzierung deutlich. Weiterhin wird in den gewählten Beschreibungskategorien ein Funktionsanspruch deutlich, der 
sich aus einer anwendungsorientierten Perspektive ergibt. Probleme, Lösungen, gewünschte Ziele, Aufgaben, Ergebnisse und Evaluationen, um einige der Elementbeschreibungen aufzunehmen, entspringen terminologisch einem betriebswirtschaftlichen bzw. praxisbezogenem Gestaltungsanspruch. Den Forschungsarbeiten zu externem und internem Crowdsourcing ist denn auch gemein, dass sie einen stark innovationszentrierten Ansatz verfolgen, der mit volks- bzw. betriebswirtschaftlich geprägten Beschreibungskategorien einhergeht (Ebner et al. 2009; Keinz 2015; Garcia Martinez 2017; Palin und Kaartemo 2016; Zhu et al. 2014, 2016; Zuchowski et al. 2016; Thuan 2019). Dies liegt auch daran, dass es sich bei vielen Forschungsprojekten im Kontext von (internem) Crowdsourcing um ,,angewandte Wissenschaft“ handelt, die im Verbund vor allem auch wirtschaftliche Perspektiven in den Blick nehmen (Blohm et al. 2018; Zhu et al. 2016; Thuan 2019). Hierin begründet sich eine Eigenlogik für die theoretischen Systembeschreibung von IC, mit dem Ergebnis, dass das Erkennen und Verstehen sich auf zielgesteuerte und organisationale Praxis, Koordinationsmechanismen und Organisationsmuster, Steuerungs- und Führungsmechanismen sowie Managementimplikationen zur Innovationsgenerierung konzentriert.

Für Pedersen et al. (2013), Zogaj et al. (2014) und Zuchowski et al. (2016) scheint klar, dass sich dieser anwendungsorientierte, zielgeleitete Steuerungsaspekt von CrowdsourcingAktivitäten für die Beschreibung einer IC-Systematik eignet und sich als Governance überschreiben lässt. Bei Pedersen et al. (2013, S. 582) heißt es hierzu: „Governance is the actions and policies employed to effectively manage the crowd and steer them toward the desired solution."

Im weiteren Verlauf formulieren Pedersen et al. fünf Governance-Herausforderungen („Effective task break-down mechanism“, „Effective task integration mechanismen“, „Effective incentive mechanism“, „Effective quality assurance system“) und fünf Governance-Mechanismen (,Right Incentive Mechanism“, „Managing Submissions“, „Loss of Control“", ,Quality of the Ideas“, „Creating Trust“). Warum Herausforderungen als Mechanismen beschrieben und Mechanismen mit bspw. „Loss of Control“ als Herausforderungen zusammengefasst sind, scheint einer fehlerhaften Tabellenüberschrift geschuldet, lässt sich letztendlich aber nicht nachvollziehen. In diesem Sinne erschließt sich bei Pedersen et al. (2013) eine vertiefte Governance-Definition nicht über das aufgeführte Zitat hinaus.

Zogaj und Bretschneider (2014) nähern sich der Governance-Problematik indem sie die Implementation von Crowdsourcing anhand von drei Praxisbeispielen analysieren, um insbesondere Informationen in Hinblick auf die von Governance-Mechanismen zu erhalten. Laut Dahlander et al. (2008, S. 118) sind Governance-Mechanismen maßgebend für den Charakter und die Qualität der Teilnahme an verschiedenen „Online-Communities“ zur Förderung und Generierung von Innovation. In diesem Sinne beziehen Zogaj und Bretschneider (2014, S. 4) diese Mechanismen auf die Durchführungs- und Handlungsebene, denn , ,[...] governance is carried out by means of different mechanisms, so-called governance mechanisms“" (Dahlander et al. 2008). 
Zur Beschreibung von Governance selbst übernehmen sie im Wesentlichen eine Definition von Markus (2007, S. 152), die erkennbar auf einem Zitat von Lynn et al. (2001, S. 6) beruht. Zogaj und Bretschneider (2014, S. 4) definieren Governance bei Crowdsourcing dann als

[...] means of achieving the direction, control and coordination of wholly or partially autonomous individuals on behalf of a crowdsourcing initiative to which they (jointly)contribute.

Während Zogaj und Bretschneider ihre Definition auf Markus aufbauen, der diese für den Kontext von Open Source Software adaptiert (2007, S. 152), verweist Markus wiederum auf Lynn et al., die für ihren Definitionsrahmen ausdrücklich auf ,public-sector applications“" (2001, S. 5) verweisen. Damit gibt es an dieser Stelle einen ersten Hinweis auf die Ursprünge des fachwissenschaftlichen und theoretischen Bezugsrahmens in der Anwendung des Governance-Konzeptes bei Crowdsourcing. Denn die Beschäftigung mit ,publicsector applications“" ist ein originär politik- und verwaltungswissenschaftlicher, auch sozialwissenschaftlicher, Forschungsfokus.

Zuchowski et al. (2016, S. 171), die explizit auf das Definitionsangebot von Pedersen et al. (2013) und Zogaj und Bretschneider (2014) verweisen, führen das steuerungs- und managementbasierte Verständnis in ihrem Definitionsangebot fort: „,We understand as ,governance “ all actions and policies used to govern, manage, and steer the crowd and internal crowdsourcing."

Sie führen ergänzend die Kategorie ,crowdsourcing governance tasks“ ein, die sechs Aufgaben- und Handlungsfelder für Governance beschreibt. Einige dieser Handlungsfelder werden dabei exklusiv für internes Crowdsourcing angenommen. Dies gilt insbesondere für die erste Kategorie „(a) management of corporate culture and change“. Die weiteren Kategorien werden überschrieben mit ,(b) incentive design; (c) task definition and decomposition; (d) quality assurance; (e) community management; and (f) management of regulations and legal implications“" (Zuchowski et al. 2016, S. 171 f.). Zusammenfassend stellen Zuchowski et al. (2016, S. 172) für die Beschreibung der einzelnen ,governance tasks“ fest, dass ,,[...] the above discussion shows important differences between governance of internal crowdsourcing and external crowdsourcing and hierarchy-based work."

Damit wird an dieser Stelle eine funktionale Differenzierung von Governance bei Crowdsourcing vorgenommen, die sich am Geltungsbereich externes im Unterschied zu internes Crowdsourcing orientiert. Diese Notwendigkeit zur Differenzierung wird auch von Knop et al. (2017, S. 2) herausgearbeitet und mit strukturellen Unterschieden zwischen externem und internem Crowdsourcing begründet.

Als abschließendes Definitionsangebot sei auf Blohm et al. (2018, S. 7) verwiesen, die auf Grundlage einer Analyse von Governance-Mechanismen an 19 Fallbeispielen zu folgendem Verständnis gelangen:

In crowdsourcing, governance involves structuring roles and responsibilities, formal and informal rules, standards and regulations, outcome control measures, communication processes, or matters of task allocation in order to achieve the crowdsourcer's goal. 
Hierauf aufbauend definieren Blohm et al. (2018, S. 7 f.) sechs Klassen - ,Task Definition“, „Task Allocation“, „Quality Assurance“, „Incentives“, ,Qualification“ und „Regulation" - innerhalb derer sich 21 Governance-Mechanismen verorten und beschreiben lassen. Der Verweis der Autoren auf vorangegangene Studien (2018, S. 8), die zu einer eindeutigen Identifikation dieser Mechanismen geführt haben, kann nicht nachvollzogen werden, denn: „In order to ensure the possibility of a blind review, we do not cite these studies."

Entsprechend der übergeordneten Fragestellung dieses Beitrages, lassen sich bereits einige Teilkategorien und Aspekte für die Beschreibung eines Crowdsourcing-Systems herausarbeiten. (1) Zunächst können Modell-Elemente bzw. Komponenten für eine Crowdsourcing-Konzeption identifiziert werden (Pedersen et al. 2013; Zuchowski et al. 2016), die sich, so schlagen es Knop et al. (2017) vor, aus der Perspektive soziotechnischer Systeme gut beschreiben lassen. (2) Allen Zugängen ist gemein, dass sie einen Steuerungsanspruch unterstellen, der sich dem Ziel unterordnet, eine ,desired solution“ (Pedersen et al. 2013, S. 582) bzw. ,the crowdsourcer's goal“ (Blohm et al. 2018, S. 7) zu erzielen. Um dies zu erreichen, wie oben beschrieben und hinreichend zitiert, bedarf es (3) der Steuerung in Form einer Governance. Die Governance wiederrum lässt sich (4) in einzelne Mechanismen (Zogaj et al. 2014; Blohm et al. 2018) bzw. ,crowdsourcing governance tasks“ (Zuchowski et al. 2016, S. 171) unterteilen. (5) Funktional und strukturell ist zwischen externem und internem Crowdsourcing, auch in der Anwendung von GovernanceMechnismen, zu unterscheiden (Zuchowski et al. 2016; Knop et al. 2017).

\subsection{In der Governance-Falle?}

Diese erste Ordnung vorgeschlagener Beschreibungskategorien kann aber nur als erster Strich schemenhafter Skizzierungsabsichten verstanden werden. Wenn es das Ziel ist zu definitorischer und begrifflicher Eindeutigkeit zu kommen, um vor dem Hintergrund eines ordnenden Gesamtverhältnisses sinnvoll referenzieren und Besagtes in einen zielführenden Wissenschaftsdiskurs überführen zu können, fehlen Klarheit und Verbindlichkeit. Wenn mit „Structure“ bei Knop et al. (2017, S. 3) Kommunikationssysteme, Autoritätssysteme und Workflow-Systeme gemeint sind, die sowohl normative Dimension (Werte, Normen und allgemeine Rollenerwartungen) als auch Verhaltensdimension (Verhaltensmuster, Kommunikation, Autorität) beinhalten und der Governance-Begriff bei Knop et al. (2017, S. 7) die Strukturierung von Rollen und Verantwortlichkeiten, formalen und informellen Regeln, Normen und Vorschriften, Maßnahmen zur Ergebniskontrolle, Kommunikationsprozesse oder Fragen der Aufgabenverteilung beinhaltet, um das Ziel des Crowdsourcers zu erreichen, stellt sich die Frage, was ist denn nicht gemeint? Die Breite der vorgeschlagenen Definitionen mach es darüber hinaus schwierig herauszuarbeiten, wie sich das Eine vom Anderen unterscheidet.

Natürlich ist das Ziel eine im Erstzugang möglichst umfassenden Definition zu formulieren verständlich und bei der Erschließung neuer Phänomene geradezu notwendig. Es ist 
im grundsätzlichen Forschungsverlauf jedoch angezeigt, immerhin wünschenswert, die anfängliche Unschärfe zu neuer Klarheit zu verdichten. Wie in der Einleitung bereits erwähnt, kann es hier sinnvoll sein, interdisziplinäre Zugänge zu wählen, um zu prüfen, ob sich Bestandswissen, etwa in Form vorhandener Theorie- und Terminologieangebote, eignet, im vorliegenden Fall zur Anwendung zu kommen. Vordringlich geboten scheint an dieser Stelle eine Auseinandersetzung mit der Bedeutung und dem Anwendungsbereich von Steuerungskategorien bei Crowdsourcing. Interessant sind dabei die Beziehungen zwischen Steuerungssubjekt und Steuerungsobjekt, wie sie in den Governance-Definitionen zu Crowdsourcing Einzug finden. Insbesondere gilt es aber die Bedeutung und den Geltungsbereich der Terminologie Governance selbst zu fassen. Da der einzig substanzielle Verweis in Bezug auf Governance mit Lynn et al. (2001) in Richtung Politikwissenschaften deutet, soll in Folgendem das Definitionsangebot innerhalb dieser Disziplin beleuchtet und auf Übertragbarkeit geprüft werden.

\subsubsection{Governance in der Politikwissenschaft}

Das Konzept der Governance zählt laut Peters (2010, S. 2) zu einem der meist Verwendeten, gar als „Fetisch“ bezeichneten, Modebegriffe der Politikwissenschaft der letzten Jahre. Dabei begründet sich in der Mehrdeutigkeit des Konzeptes sein Erfolg, denn (Peters 2010, ebd.):

[...] it can be shaped to conform to the intellectual preferences of the individual author and therefore to some extent obfuscates meaning at the same time that it perhaps enhances understanding.

Der Anwendungsbereich des Governance-Begriffs lässt sich durch ergänzende Attribute und Nomen dabei fast beliebig erweitern (Offe 2009, S. 557). Zu lesen ist von ,sectoral governance“, „good governance“, „corporate governance“, ,public governance“, „multi-level governance“, ,sustainable governance“, „,global governance“, ,,environmental governance“, ,,cultural governance“, ,,earth system governance“ oder auch ,,polycentric governance in telecoupled resource systems“ um nur einige Beispiele zu nennen (Biermann et al. 2019; Newig et al. 2019; Brunnengräber et al. 2004; Grande 2012). Ist Governance also die Allzweckwaffe für jede Art von (gesellschaftlichen) Regelungsproblem, wie Grande (2012, S. 566) vermutet? Tatsächlich lassen sich, obwohl es sich um einen ,annerkannt uneindeutingen Begriff“ handelt, grundsätzliche Gemeinsamkeiten in den Charakteristika immerhin der Anwendungsbereiche von Governance identifizieren (Blumenthal 2005; Lembcke et al. 2016).

Klar ist, dass es sich bei der Begrifflichkeit „Governance“ um mehr handelt als einen Anglizismus, auch wenn oftmals die gleichen Themen unter den verschiedenen Leitbegriff „Steuerung“ und „Governance“ behandelt werden (Mayntz 2004). Eine gleichbedeutende Verwendung würde implizieren, dass auch im Englischen unter ,governing“ und 
„governance“ das gleiche zu verstehen sei. Allerdings ist die Zusammenfassung von Fasenfest (2010, S. 771), wonach „Government: the office, authority or function of governing “, „Governing: having control or rule over oneself“" und „Governance: the activity of governing“ bedeutet, unzutreffend. Tatsächlich wird „governance“ und „governing“ im Unterschied zur historischen Anwendung auch im englischen Sprachgebrauch heute nicht mehr gleichbeutend verwendet und lässt sich nicht synonym im Sinne einer hierarchiegebundene Steuerung, die sich ursprünglich auf den Prozess des Regierens bezieht, anwenden (Mayntz 2008, S. 45). Ganz im Gegenteil meint Governance, so wird zu zeigen sein, Steuerung als Kooperation und Koordination.

Damit wird im Ansatz deutlich, dass Governance-Konzepte in der Politikwissenschaft mehr sind als ein „Empty Signifier“ (Offe 2009). Laut Grande (2012, S. 566 f.) gibt es einen konzeptionellen Kern, der sich als gemeinsamer Nenner der vielfältigen Ansätze beschreiben lässt und anhand von fünf Merkmalen zusammengefasst werden kann:

Das erste und wichtigste Merkmal ist die Betonung nicht-hierarchischer Formen der Produktion öffentlicher Güter.

Damit verbunden ist, zweitens, eine Kritik am Staat als dem exklusiven Produzenten öffentlicher Güter. Für die Governance-Konzepte charakteristisch ist [...], dass nicht-staatliche Akteure und Organisationen [...] an Bedeutung gewinnen.

Diese Kritik an Hierarchien als Steuerungsprinzip und die Einbeziehung privater Akteure in die Produktion öffentlichen Güter wird, das wäre das dritte gemeinsame Merkmal, als eine notwendige Folge von Interdependenz interpretiert.

[...] Aufgrund dieser zunehmenden Interdependenz, aber auch infolge des Bedeutungsverlusts territorialer und funktionaler Handlungsgrenzen ist, viertens, die Komplexität politischen Handelns erheblich gestiegen.

[...] All dies hat schließlich zur Folge, dass die Notwendigkeit und die Bedeutung von Kooperation und Koordination zwischen verschiedensten Akteuren erheblich zunehmen.

Ihren empirischen Ursprung finden die Governance-Konzepte unter anderem in der Beobachtung zunehmender Interdependenzen zwischen gesellschaftlichen Teilsystemen und territorialen Handlungsebenen (Grande 2012, ebd.). In der zweiten Hälfte des letzten Jahrhunderts geriet das Verständnis einer traditionellen Konzeptualisierung des öffentlichen Sektors, wonach der Staat als wichtigster Akteur Einfluss nimmt auf Wirtschaft und Gesellschaft, unter Druck. Ein Teil der Belastung für die nationalen Regierungen ist das Ergebnis der gestiegenen Bedeutung des internationalen Umfelds und einer verminderten Fähigkeit dieser Regierungen, ihre Volkswirtschaften und Gesellschaften vor dem globalen Druck zu schützen bzw. globale Herausforderungen alleine zu bewältigen (Peters und Pierre 1998, S. 223). Daneben verändern sich die Arenen und Strukturen von Aushandlungsprozessen in Form veränderter Dialogansprüche und Dialogmöglichkeiten zwischen Staat und Gesellschaft, Staat und Wirtschaft, staatlichen und nicht-staatlichen Organisationen und nationalen und supranationalen bzw. internationalen Institutionen. Als Beispiel hierfür wird immer wieder die Europäische Union angeführt, die die klassische Einheit von Recht und Politik im Nationalstaat in Frage stellt und zu einem Mehrebenensystem mit vielfältigsten Akteurskonstellationen und institutionellen Architekturen führt 
(Mayntz 2008; Brunnengräber et al. 2004; Grimm 2001; Peters und Pierre 1998). Governance in diesem Sinne beschreibt dann sowohl Akteurskonstellationen als auch Regelungsweisen (Mayntz 2008, S. 46).

Ein für die Gegenstand dieses Artikels wesentlicher Beitrag zur Governance-Forschung ist das Argument, dass es sich in der Entwicklung hin zur Governance um eine Dreischritt von der „Planung“ über die „Steuerung“ zur „Governance“ handelt (Schuppert 2016; Mayntz 2008; Grande 2012). Stand zunächst die Planung im Zentrum eines alle gesellschaftlichen Abläufe aktiv steuernden Staates, wurde die Planungssemantik als bald von einer Steuerungssemantik ersetzt und in eine Steuerungstheorie überführt (Schuppert 2016, S. 151). In der Steuerungstheorie bildet das Konzept hierarchischer Steuerung den analytischen Rahmen (Mayntz 2008, S. 43):

Dieses Konzept erlaubte es, klar zwischen Steuerungssubjekt und Steuerungsobjekt zu unterscheiden; Steuerungsobjekt sind gesellschaftliche Teilsysteme bzw. Gruppen, deren Verhalten in eine bestimmte Richtung gelenkt werden soll.

Wenn die zentrale Annahme der Steuerungstheorie also die Existenz eines Steuerungssubjektes ist, dann begreift man Governance als etwas von Steuerung grundlegend verschiedenes. In Governance-Regimen, so skizziert Grande (2012, S. 581),

[...]gibt es keinen Akteur mehr, der als autonome Steuerungsinstanz des Gesamtprozesses fungieren könnte - weder real noch imaginär. [...] Wenn es eine solche Steuerungsinstanz aber nicht mehr gibt, dann macht es auch keinen Sinn mehr, von Steuerung zu sprechen - und in den Fällen, in denen es eine solche Steuerungsinstanz noch gibt, sollte nicht von Governance gesprochen werden.

Selbst eine nicht-hierarchische Beziehung von Staat und Gesellschaft wird im Sinne der deutschen Steuerungstheorie als „Steuerung“ begriffen (Grande 2012, S. 584). Wesentlich bleibt die intentionale Steuerung durch ein Steuerungssubjekt. Auch wenn Governance definiert werden kann als ,absichtsvolle Regelung gesellschaftlicher Sachverhalte“ (Mayntz 2008, S. 55) bleibt eben offen, wie ,,in komplexen, dynamischen GovernanceStrukturen noch sinnvoll von Steuerungsintentionen“ (Grande 2012, S. 581) gesprochen werden kann.

\subsubsection{Zwischenfazit}

Überträgt man das hier dargelegte Verständnis von Governance auf den Anwendungsfall Crowdsourcing, ergeben sich einige Probleme. Tatsächlich ist die Steuerungsintention ein wesentliches Merkmal nicht nur des Crowdsourcing-Prozesses an sich, sondern - wichtiger noch - der Beschreibungsabsicht, für die das Wort Governance verwendet wird. Wie bereits festgestellt, ist allen Beschreibungsansätzen zu Crowdosurcing gemein, dass sie einen Steuerungsanspruch unterstellen, der sich dem Ziel unterordnet, eine ,desired solution“ (Pedersen et al. 2013, S. 582) bzw. ,the crowdsourcer's goal“ (Blohm et al. 2018, 
S. 7) zu erzielen. Funktional können die Beteiligten beim Crowdsourcing dabei immer in zwei Rollen aufgeteilt werden: dem „Crowdsourcer“ und dem „Crowdsourcee“. Der „Crowdsourcer“ ist der vorstehende Auftraggeber, der eine Lösung für ein gegebenes Problem sucht, die Crowdsourcees sind die Mitglieder der Crowd, die eine Lösung erarbeiten sollen (Leimeister et al. 2015).Dies gilt sowohl für externes als auch internes Crowdsourcing, die beide in diesem Zusammenhang als geschlossenes System zu betrachten sind. Damit fügen sie sich idealtypisch ein in das Verständnis von Steuerungssubjekt („Crowdsourcer“) und Steuerungsobjekt („,Crowdsourcee“, als Gruppe deren Verhalten in eine bestimmte Richtung gelenkt werden soll), wie es der Steuerungstheorie zugrunde liegt. Folgerichtig heißt es dann in der Governance-Definition für Crowdsourcing (Pedersen et al. 2013, S. 582): „Governance is the actions and policies employed to effectively manage the crowd and steer them toward the desired solution."

Damit wird deutlich, dass Governance zur Beschreibung von Crowdosurcing-Systemen eigentlich Steuerung meint. Im Sinne eines politikwissenschaftlichen Gebrauches lässt sich diese Terminologie im gegebenen Fall dann nicht sinnvoll übertragen, weil, bei aller Vielfalt der Governance-Anwendungen, explizit etwas anderes gemeint ist (s. o.).

$\mathrm{Da}$ festgestellt wurde, dass die Forschungsarbeiten $\mathrm{zu}$ externem und internem Crowdsourcing einen stark innovationszentrierten Ansatz verfolgen, der auf eine vornehmliche Beschäftigung durch volks- bzw. betriebswirtschaftlich Wissenschaftsdisziplinen hinweist, soll an dieser Stelle geprüft werden, ob in der interdisziplinären GovernanceForschung, hier insbesondere der Ökonomie, weiterführende Definitionsangebote aufgebracht werden, die übernommen werden können.

\subsubsection{Governance in der Ökonomie}

An dieser Stelle sei auf zwei Governance-Konzepte bzw. Aspekte in der Ökonomie eingegangen. Zum einen die Konzeption von „Coroporate Governance“ und theoretische Ansätze für „Economic Governance“. Während innerhalb der Ersteren Debatten im Kontext von einzelnen Unternehmen skizziert werden, zielt der zweite Begriff auf gesamtwirtschaftliche Zusammenhänge (Brunnengräber et al. 2004, S. 22). Für Lindberg et al. (1991, S. 5 f.) ist „Economic Governance“ als Phänomen auf der Mesoebene beschreibbar, d. h. in industriellen Sektoren. Governance lässt sich dort betrachten als

[...] a matrix of interdependent social exchange relationships, or transactions, that must occur among organizations, either individually or collectively, in order for them to develop, produce, and market goods and services. Thus, governance is an extremely complex phenomenon.

Damit wird auch hier, wie im politikwissenschaftlichen Zugang, der Aspekt der Interdependenz in den Vordergrund gerückt (Brunnengräber et al. 2004, S. 24). Ziel hier ist naturgemäß nicht die gemeinwohlorientierte, absichtsvolle Regelung gesellschaftlicher Sachverhalte, sondern die Entwicklung, Produktion und Vermarktung von Waren und Dienstleitungen (Lindberg et al. 1991, S. 6; Mayntz 2008, S. 45 f.). 
Unter „Corporate Governance“ werden verschiedene Rahmengesetzgebungen und Berichtspflichten als rechtlicher und faktischer Ordnungsrahmen subsumiert, es geht im Ansatz aber auch um die Frage von - verantwortungsbewusster, nachhaltiger, auf langfristige Wertschöpfung ausgerichteter - Unternehmensführung und Kontrolle (World Bank 1996, S. XIV; Brunnengräber et al. 2004, S. 7). Laut Bainbridge (2002, S. 15) haben alle Konzepte zur „Corporate Governance“ eins gemein:

They strive to answer two basic sets of questions: (1) As to the means of corporate governance, who decides? In other words, when push comes to shove, who ultimately is in control? (2) As to the ends of corporate governance, whose interests prevail? When the ultimate decisionmaker is presented with a zero sum game, in which it must prefer the interests of one constituency class over those of all others, which constituency wins?

Im Kern geht es also auch bei „Corporate Governance“ um Probleme von Steuerungsintentionen und Hierarchien in einem Mehrebenensystem. Dies wird insbesondere am Beispiel von Aktiengesellschaften, bei Shareholderansprüchen und multinational agierenden Unternehmen mit einer Vielzahl von regulatorischen und territorialen Handlungsebenen deutlich. Auch wenn die betriebliche Verfasstheit der Unternehmen eher an die Einheit von Recht und Politik in der Souveränität des Nationalstaates im späten 19. und frühen 20. Jahrhundert erinnert, gilt auch für Unternehmen, dass die Arenen und Strukturen von Aushandlungsprozessen starken Veränderungen unterworfen sind. Schlussendlich bezieht sich der Begriff der „Corporate Governance“ damit nicht auf die Binnenordnung, d. h. die Unternehmensverfassung, sondern adressiert Problemstellungen im Rahmen der Einbindung des Unternehmens in sein Umfeld (Werder 2018).

Zusammenfassend lässt sich festhalten, dass - auch wenn die Governance-Forschung in der Ökonomie deutlich weniger ausgeprägt ist und für beide Disziplinen heterogene Konzeptansätze festzustellen sind - die traditionelle Trennung der Disziplinen in politische und ökonomische Teilsysteme auch im Bereich der Governance-Forschung verschwimmt (Brunnengräber et al. 2004, S. 24):

So werden zunehmend Markt, Staat und Gesellschaft in Beziehung zueinander gesetzt, indem auf die Interdependenzen und komplexen Wechselwirkungen zwischen den gesellschaftlichen Bereichen verwiesen wird. Bedeutsam sind in den politik- wie in den wirtschaftswissenschaftlichen Konzepten die verschiedenen Handlungsebenen und -systeme (Multi-Level Governance). Die Berücksichtigung einer Vielzahl an Akteuren und Interaktionsformen wird gleichermaßen thematisiert wie die Problemkonstellationen, die sich zunehmend global ausdifferenzieren.

\subsection{Lehren aus der Governance-Forschung}

Für die vorliegende Diskussion bedeutet dies, dass auch aus den wirtschaftswissenschaftlichen Disziplinen kein Definitionsangebot für Governance vorgelegt wird, welches die vorgeschlagene Anwendung des Begriffs Governance für die Beschreibung von 
Steuerungsaktivitäten bei Crowdsourcing-Systemen angebracht erscheinen lässt. Tatsächlich eröffnet sich in diesem Zusammenhang eine Perspektive, die es notwendig macht, den Begriff in seiner politikwissenschaftlichen und wirtschaftswissenschaftlichen Bedeutung für einen späteren Anwendungsfall in der Beschreibung von Crowdsourcing zu reservieren.

Wenn Governance-Konzepte, als Modelle neuer kooperativer Netzwerksteuerung, Ausdruck ,langfristiger struktureller Veränderungen in den Modi der Produktion kollektiver Güter in modernen Gegenwartsgesellschaften sind" (Grande 2012, S. 585; Mayntz 2008, S. 46), dann liegt es nahe, Crowdsourcing selbst als Ausdruck dieser strukturellen Veränderung in den Blick zu nehmen. Dann wird Crowdsourcing zum Indikator einer systemischen Transformation durch Digitalisierung, in der internes Crowdsourcing möglicherweise Veränderungen der innerbetrieblichen Organisation und externes Crowdsourcing möglicherweise die Verlagerung der Arbeit aus der klassischen Betriebsorganisation beschreibt. Wenn ein solcher Transformationsprozess - was aktuell (noch) nicht zu beobachten ist - zu einer Situation führt, in der die Rollen „Crowdsourcer“ und „Crowdsourcee“ nicht mehr im steuerungstheoretischen Sinn einem Subjekt und Objekt zugeordnet werden können, durch Crowdsourcing also die strukturelle Voraussetzung für ,relativ autonome, funktionelle Teilsysteme“ (Mayntz 2008, S. 48) geschaffen werden, dann ist es nicht nur sinnvoll, sondern notwendig, das Governance-Konzept an dieser Stelle im Sinne einer Crowd-Governance einzuführen.

In ähnlicher Weise schlagen Fenwick et al. (2018, S. 9), wenn auch aus einer juristischen, marktregulatorischen Perspektive, die Weiterentwicklung der „Corporate Governance“ hin zu einer „Platform Governance“ vor.

Given the proliferation of platforms, we seem to be living through a shift from a world of firms to a new world of platforms. In the same way that the "firm" came to replace ,contracts" for many business activities in the context of the industrial revolution, ,platforms“ are now replacing ,oldworld firms“ in the context of the digital transformation.

Um den sich hieraus ergebenden Herausforderungen für die Wirtschaft zu begegnen, ist es laut Fenwick und Vermeulen (2019, S. 2) notwendig, zeitgemäße Richtlinien und Vorschriften im Sinne einer „Corporate Governance“ als „Plattform Governance“ zu entwickeln.

In Bezug auf den Einfluss von Crowdsourcing auf die betriebliche Verfasstheit, ihre Organisation und die Arbeitssubjekte wurden im Rahmen von ICU Annahmen formuliert, die eine ähnliche Entwicklung antizipieren (Otte und Schröter 2018, S. 5):

Durch das schrittweise Zusammenwachsen virtueller Transaktionsräume und bislang eigenständiger Plattformen entfalten sich neue Potenziale des Crowdsourcings als Teil modernen Crowdworkings. Das auftragsbezogene Denken und Handeln wie auch die Orientierung an nicht mehr nur vertikalen sondern zukünftig vor allem horizontalen Wert-schöpfungsketten wird im Verbund mit Modellen des teilautonomen sowie agilen Arbeitens zur dominierenden Kultur der Crowdsourcing-Anwendungen. Die Grenzen zwischen internem und externem Crowdsourcing beginnen, sich zu verwischen. Beide Dynamiken gehen ineinander über. Durch die voranschreitende Entgrenzung der Betriebsverfasstheit heben sich interne und externe Nutzungen wechselseitig auf. 
Um solche Realitäten bei Crowdsourcing systemtheoretisch abbilden und beschreiben zu können, bietet sich dann ggf. der Governance-Begriff an, wie er in den letzten 50 Jahren vor allem durch die Politikwissenschaft geprägt wurde.

\subsection{Fazit}

Im Ergebnis geht damit die deutliche Empfehlung einher, den Governance-Begriff, so wie er bei Crowdsourcing in den Definitionen von Pedersen et al. (2013), Zogaj et al. (2014), Zuchowski et al. (2016) und Blohm et al. (2018) zur Anwendung kommt, durch das Wort „Steuerung“ im deutschen bzw. „Management“ im englischen Sprachgebrauch zu ersetzten. In den vorgeschlagenen Definitionen kann nicht sinnvollerweise von Governance gesprochen werden.

Weiterhin bedeutet dies, auch in den von Pedersen et al. (2013) und Zuchowski et al. (2016) entwickelten Modell-Elementen bzw. Komponenten einer CrowdsourcingKonzeption sollte das Wort „Governance“ ersetzt werden. Dies gilt konsequenterweise auch für die Beschreibung von ,governance mechanisms“ (Pedersen et al. 2013; Blohm et al. 2018) oder „,crowdsourcing governance tasks“ (Zuchowski et al. 2016). In Bezug auf die Beschreibung von Elementen einer Crowdsourcing-Konzeption sei an dieser Stelle auf Knop et al. (2017) verwiesen, die mit der Kategorie „Structures“ ein Beschreibungsangebot vorlegen, das sich möglicherweise in diesem Sinnzusammenhang einführen lässt und sich vor dem Hintergrund der hier gegenständlichen Problemlage besser eignen würde.

Schlussendlich sei darauf hingewiesen, dass es nicht darum geht eine Begrifflichkeit „nur" deshalb zu ersetzten, weil eine andere sich besser eignet. Viel wichtiger wird angenommen, dass in der zukünftigen Beschreibung einer Crowdsourcing-Systematik die Notwendigkeit zur Beschreibung einer Crowdosurcing-Governance und hiervon ausgehender Governance-Mechanismen im Sinne des politik- und wirtschafswissenschaftlich geprägten und dargelegten Verständnisses besteht. Daher sollte die Begrifflichkeit nicht zur Beschreibung von Steuerungsprinzipien und -mechanismen eingeführt werden, was nicht nur in einer interdisziplinären Betrachtung zu Missverständnissen führen muss, sondern auch in einer zukünftigen Theoriebildung zu Crowdsourcing.

Im Sinne der übergeordneten Fragestellung sollten dann die hier beschriebenen definitorischen Änderungen und Ergänzungen vorgenommen werden. Wenngleich dies ein erster Schritt in Richtung sinnvoller Begriff-Referenzierung ist, konnte die Ordnung eines Gesamtverhältnisses von Teilkategorien und Aspekten zur Beschreibung einer Crowdsourcing-Systematik damit nur ansatzweise vorbereitet werden.

In Bezug auf die Frage, wie und ob bereits beschriebene Teilkategorien und Aspekte eines IC-Systems sinnvoll referenziert und in ein ordnendes Gesamtverhältnis gesetzt werden können, muss festgestellt werden, dass sich die vorgeschlagene Beschreibungskategorien aufgrund ihrer extrem breiten Definitionen nur bedingt vergleichen und folglich ordnen lassen. Hieraus ergibt sich ein wesentlicher und notwendiger Theoretisierungsbedarf für die wissenschaftliche Auseinandersetzung mit Crowdsourcing. 


\section{Literatur}

Alam, S. L., \& Campbell, J. (2013). Role of relational mechanisms in crowdsourcing governance: An interpretive analysis. https://www.researchgate.net/publication/288206525. Zugegriffen am 18.02.2019.

Bainbridge, S. M. (2002). Director primacy: The means and ends of corporate governance. SSRN Journal. https://doi.org/10.2139/ssrn.300860.

Baxter, G., \& Sommerville, I. (2011). Socio-technical systems: From design methods to systems engineering. Interacting with Computers, 23, 4-17. https://doi.org/10.1016/j.intcom.2010.07.003.

Beese, J., Kazem, H., \& Aier, S. (2015). On the conceptualization of information systems as sociotechnical phenomena in simulation-based research. https://pdfs.semanticscholar.org/63ed/ 4e594c732636f2b52327efee89fd288b557e.pdf. Zugegriffen am 30.10.2018.

Biermann, F., Betsill, M. M., Burch, S., Dryzek, J., Gordon, C., Gupta, A., Gupta, J., Inoue, C., Kalfagianni, A., Kanie, N., Olsson, L., Persson, Å., Schroeder, H., \& Scobie, M. (2019). The Earth System Governance Project as a network organization: A critical assessment after ten years. Current Opinion in Environmental Sustainability, 39, 17-23. https://doi.org/10.1016/j.cosust.2019.04.004.

Blohm, I., Zogaj, S., Bretschneider, U., \& Leimeister, J. M. (2018). How to manage crowdsourcing platforms effectively? California Management Review, 60, 122-149. https://doi.org/10.1177/ 0008125617738255 .

Blumenthal, J. (2005). Governance - eine kritische Zwischenbilanz. Zeitschrift für Politikwissenschaft, 15, 1149-1180.

Brunnengräber, A., Dietz, K., \& Hirschl, B., \& Walk, H. (2004). Interdisziplinarität in der Governance-Forschung. https://www.ioew.de/publikation/interdisziplinaritaet_in_der_governance_forschung/. Zugegriffen am 19.09.2019.

Dahlander, L., Frederiksen, L., \& Rullani, F. (2008). Online communities and open innovation. Industry and Innovation, 15, 115-123. https://doi.org/10.1080/13662710801970076.

Ebner, W., Leimeister, J. M., \& Krcmar, H. (2009). Community engineering for innovations: The ideas competition as a method to nurture a virtual community for innovations. $R \& D$ Management, 39, 342-356. https://doi.org/10.1111/j.1467-9310.2009.00564.x. Zugegriffen am 19.09.2019.

Fasenfest, D. (2010). Government, governing, and governance. Critical Sociology, 36, 771-774. https://doi.org/10.1177/0896920510378192.

Fenwick, M., \& Vermeulen, E. P. M. (2019). A sustainable platform economy \& the future of corporate governance. SSRN Journal. https://doi.org/10.2139/ssrn.3331508.

Fenwick, M., McCahery, J. A., \& Vermeulen, E. P. M. (2018). The end of 'corporate' governance: Hello 'platform' governance. SSRN Journal. https://doi.org/10.2139/ssrn.3232663.

Garcia Martinez, M. (2017). Inspiring crowdsourcing communities to create novel solutions: Competition design and the mediating role of trust. Technological Forecasting and Social Change, 117, 296-304. https://doi.org/10.1016/j.techfore.2016.11.015.

Grande, E. (2012). Governance-Forschung in der Governance-Falle? - Eine kritische Bestandsaufnahme. PVS, 53, 565-592. https://doi.org/10.5771/0032-3470-2012-4-565.

Grimm, D. (2001). Die Verfassung und die Politik; Einsprüche in Störfällen. München: Beck.

Keinz, P. (2015). Auf den Schultern von ... Vielen! Crowdsourcing als neue Methode in der Neuproduktentwicklung. Schmalenbachs Zeitschrift für betriebswirtschaftliche Forschung, 67, 35-69. https://doi.org/10.1007/BF03372915.

Knop, N., Durward, D., \& Blohm, I. (2017). How to design an internal crowdsourcing system. https://www.alexandria.unisg.ch/252020/1/JML_672.pdf. Zugegriffen am 19.09.2019.

Leimeister, J. M., Zogaj, S., \& Durward, D. (Hrsg.). (2015). New forms of employment and IT Crowdsourcing. 
Lembcke, O. W., Ritzi, C., \& Schaal, G. S. (Hrsg.). (2016). Zeitgenössische Demokratietheorie. Wiesbaden: Springer Fachmedien Wiesbaden.

Lindberg, L. N., Campbell, J. L., \& Hollingsworth, J. R. (1991). Economic governance and the analysis of structural change in the American economy. In J. L. Campbell, J. R. Hollingsworth \& L. N. Lindberg (Hrsg.), Governance of the American economy (S. 3-34). Cambridge: Cambridge University Press.

Lynn, L. E., Heinrich, C. J., \& Hill, C. J. (2001). Improving governance: A new logic for empirical research. Washington, DC: Georgetown University Press.

Markus, M. L. (2007). The governance of free/open source software projects: Monolithic, multidimensional, or configurational? Journal of Management and Governance, 11, 151-163. https:// doi.org/10.1007/s10997-007-9021-x.

Mayntz, R. (2004). Governance Theory als fortentwickelte Steuerungstheorie? Köln: Max-PlanckInstitut für Gesellschaftsforschung. http://hdl.handle.net/10419/44296

Mayntz, R. (2008). Von der Steuerungstheorie zu Global Governance. In G. F. Schuppert \& M. Zürn (Hrsg.), Governance in einer sich wandelnden Welt (S. 43-60). Wiesbaden: VS Verlag für Sozialwissenschaften.

Newig, J., Lenschow, A., Challies, E., Cotta, B., \& Schilling-Vacaflor, A. (2019). What is governance in global telecoupling? Ecology and Society, 24. https://doi.org/10.5751/ES-11178-240326.

Offe, C. (2009). Governance: An „empty signifier?“. Constellations, 16, 550-562. https://doi. org/10.1111/j.1467-8675.2009.00570.x.

Otte, A., \& Schröter, W. (2018). Lebende Konzernbetriebsvereinbarung als soziale Innovation; Internes Crowdsourcing in der GASAG-Gruppe Bedeutung - Bewertung - Wortlaut. http://www. blog-zukunft-der-arbeit.de/wp-content/uploads/2018/07/Lebende_KBV_Otte_Schroeter.pdf. Zugegriffen am 19.09.2019.

Palin, K., \& Kaartemo, V. (2016). Employee motivation to participate in workplace innovation via in-house crowdsourcing. European Journal of Workplace Innovation, 2, 19-40.

Pedersen, J., Kocsis, D., Tripathi, A., Tarrell, A., Weerakoon, A., Tahmasbi, N., Xiong, J., Deng, W., Oh, O., \& de Vreede, G.-J. (2013). Conceptual foundations of crowdsourcing: A review of IS Research 2013 46th Hawaii International Conference on System Sciences. IEEE, S. 579-588.

Peters, B. G., \& Pierre, J. (1998). Governance without government? Rethinking public administration. Journal of Public Administration Research and Theory, 8, 223-243. https://doi.org/10.1093/ oxfordjournals.jpart.a024379.

Peters, G. (2010). Governance as political theory. http://regulation.huji.ac.il/papers/jp22.pdf. Zugegriffen am 19.09.2019.

Schuppert, G. F. (2016). Governance in der Demokratietheorie. In O. W. Lembcke, C. Ritzi \& G. S. Schaal (Hrsg.), Zeitgenössische Demokratietheorie. Wiesbaden: Springer Fachmedien Wiesbaden.

Thuan, N. H. (2019). Business process crowdsourcing: Concept, ontology and decision support. Cham: Springer.

Werder, A. (2018). Corporate governance: Definition. https://wirtschaftslexikon.gabler.de/definition/corporate-governance-28617/version-367554. Zugegriffen am 19.09.2019.

World Bank. (1996). Governance: The World Bank's experience. Washington, DC: The World Bank.

Zhu, H., Djurjagina, K., \& Leker, J. (2014). Innovative behaviour types and their influence on individual crowdsourcing performances. International Journal of Innovation, Management and Technology, 18, 1-18. https://doi.org/10.1142/S1363919614400155.

Zhu, H., Sick, N., \& Leker, J. (2016). How to use crowdsourcing for innovation?: A comparative case study of internal and external idea sourcing in the chemical industry. In D. F. Kocaoglu (Hrsg.), Technology management for social innovation. PICMET'16: Portland International Conference on Management of Engineering and Technology: Proceedings. Piscataway: IEEE. 
Zogaj, S., \& Bretschneider, U. (2014, June 9-11). Analyszing governance mechanisms for crowdsourcing information systems: A multiple case analysis. In: M. Avital, J. M. Leimeister \& U. Schultze (Hrsg.), ECIS 2014 proceedings. 22th European Conference on Information Systems. Tel Aviv: AIS Electronic Library.

Zogaj, S., Bretschneider, U., \& Leimeister, J. M. (2014). Managing crowdsourced software testing: A case study based insight on the challenges of a crowdsourcing intermediary. Journal of Business Economics, 84, 375-405. https://doi.org/10.1007/s11573-014-0721-9.

Zuchowski, O., Posegga, O., Schlagwein, D., \& Fischbach, K. (2016). Internal crowdsourcing: Conceptual framework, structured review, and research agenda. Journal of Information Technology, 31, 166-184. https://doi.org/10.1057/jit.2016.14.

Open Access Dieses Kapitel wird unter der Creative Commons Namensnennung 4.0 International Lizenz (http://creativecommons.org/licenses/by/4.0/deed.de) veröffentlicht, welche die Nutzung, Vervielfältigung, Bearbeitung, Verbreitung und Wiedergabe in jeglichem Medium und Format erlaubt, sofern Sie den/die ursprünglichen Autor(en) und die Quelle ordnungsgemäß nennen, einen Link zur Creative Commons Lizenz beifügen und angeben, ob Änderungen vorgenommen wurden.

Die in diesem Kapitel enthaltenen Bilder und sonstiges Drittmaterial unterliegen ebenfalls der genannten Creative Commons Lizenz, sofern sich aus der Abbildungslegende nichts anderes ergibt. Sofern das betreffende Material nicht unter der genannten Creative Commons Lizenz steht und die betreffende Handlung nicht nach gesetzlichen Vorschriften erlaubt ist, ist für die oben aufgeführten Weiterverwendungen des Materials die Einwilligung des jeweiligen Rechteinhabers einzuholen.

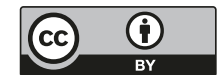

